\title{
Insuffisance hépatique chez le mouton par intoxication expérimentale par l'alcool allylique

\author{
$\mathrm{H}$ Brugère, $\mathrm{T}$ Argaw \\ École nationale vétérinaire, 94704 Maisons-Alfort, France
}

Summary - Experimental liver damage by allyl alcohol in sheep. An intravenous injection of allyl alcohol $(12 \mathrm{mg} / \mathrm{kg}$ ) to sheep induced acute hepatitis with a decreased elimination of sulfobromorphtalein, and an increase of blood ammonia and bilirubin. It is assumed that the important augmentation of conjugated bilirubin was the result of cholestasis appearing as a consequence of the inflammation in the periportal areas.

Les modèles expérimentaux d'insuffisance hépatique reproduisent des altérations morphologiques et fonctionnelles qui ne correspondent que de façon imparfaite aux modifications observées en pathologie spontanée. Le modèle d'hépatite toxique induite par le $\mathrm{CCl}_{4}$ n'échappe pas à cette critique, et c'est pourquoi la production de lésions différentes dans leur localisation a été recherchée par utilisation d'un autre toxique, l'alcool allylique, connu pour produire chez le rat des lésions périportales.

Matériel et méthodes - Dix moutons croisés île-de-France ont reçu l'alcool allylique (solution à $12 \mathrm{mg} / \mathrm{kg}$, voie IV). La dose toxique nécessaire pour produire une hépatite sublétale avait été déterminée préalablement sur d'autres animaux.

Le bilan fonctionnel hépatique des animaux a été réalisé $7 \mathrm{j}$ avant et $24 \mathrm{~h}$ après l'intoxication par le test d'épuration de la bromo-sulfone-phtaléine (BSP), la détermination des 2 formes (libre el conjuguée) de la bilirubine et la mesure de l'ammoniémie. La BSP est injectée à raison de $5 \mathrm{mg} / \mathrm{kg}$, IV, en solution à $30 \mathrm{mg} / \mathrm{ml}$ et les prélèvements sanguins effectués de façon à suivre la décroissance de la concentration plasmatjque pendant $1 \mathrm{~h}$ (prélèvements à $2,5,8,20$, $30,45 \mathrm{~min}$ ). La bilirubine est dosée par la méthode classique de Van den Bergh, et la détermination de ses différentes fractions est possible par utilisation d'un étalon (sérum de bœuf enrichi en fractions libre et conjuguee "bilirubine C et T", Biotrol, Paris). L'ammoniémie est mesurée par chimie sur réactifs secs (formation d'un complexe avec le bleu de bromophénol, lecture à $605 \mathrm{~nm}$, Kodak Ektachem DT).

En plus de ces épreuves d'exploration fonctionnelle, des biopsies sont pratiquées, soit à l'aiguille, soit après laparotomie, pour étude histologique (Argaw, 1989).

Résultats et discussion - Les 10 moutons ont survécu et présenté des signes cliniques et fonctionnels d'insuffisance hépatique, avec cependant une certaine variabilité, aussi bien dans les valeurs biochimiques que dans les lésions histologiques. Les résultats des épreuves fonctionnelles sont présentées dans le tableau I.

L'étude des tissus prélevés par biopsie a fait apparaître des lésions d'hépatite aiguë dégénérative dont le siège était la région périportale chez 6 animaux. Des lésions de stéatose ou de choléstase ont aussi été observées. L'hétérogénéité des lésions est en corrélation avec la dispersion des valeurs des paramètres d'exploration fonctionnelle.

Alors que le $\mathrm{CCl}_{4}$ et la plupart des substances hépato-toxiques (acétaminophène, chloroforme...) présentent une affinité plus marquée pour la région 
Tableau I. Valeurs des demi-vies de distribution $\left(T_{1 / 2} \alpha\right)$ et d'élimination $\left(T_{1 / 2} \beta\right)$ de la BSP, des concentrations plasmatiques de bilirubine libre (BL), conjuguée (BC), totale (BT), et de l'ammoniac, avant et après administration d'alcool allylique $(12 \mathrm{mg} / \mathrm{kg})$.

\begin{tabular}{|c|c|c|c|c|c|c|c|c|}
\hline & & \multicolumn{2}{|c|}{$B S P$} & \multicolumn{4}{|c|}{ Bilirubine } & \multirow{3}{*}{$\begin{array}{c}\mathrm{NH}_{3} \\
(\mu \mathrm{mol} / \mathrm{l})\end{array}$} \\
\hline & & $T_{1 / 2} \alpha$ & $T_{1 / 2} \beta$ & $B L$ & $B C$ & $B T$ & $B C / B T$ & \\
\hline & & \multicolumn{2}{|c|}{$(\min )$} & & $(\mu \mathrm{mol} / \mathrm{l})$ & & $(\%)$ & \\
\hline Avant & $\begin{array}{l}M \\
\sigma\end{array}$ & $\begin{array}{l}2,87 \\
0,73\end{array}$ & $\begin{array}{r}17,60 \\
6,59\end{array}$ & $\begin{array}{l}0,82 \\
0,40\end{array}$ & $\begin{array}{l}1,02 \\
0,41\end{array}$ & $\begin{array}{l}1,85 \\
0,69\end{array}$ & $\begin{array}{l}56,21 \\
10,04\end{array}$ & $\begin{array}{r}45,4 \\
8,2\end{array}$ \\
\hline Après & $\begin{array}{l}M \\
\sigma\end{array}$ & $\begin{array}{r}13,52 \\
4,27\end{array}$ & $\begin{array}{l}56,68 \\
20,13\end{array}$ & $\begin{array}{l}5,01 \\
5,37\end{array}$ & $\begin{array}{l}9,34 \\
8,08\end{array}$ & $\begin{array}{l}14,36 \\
13,36\end{array}$ & $\begin{array}{l}69,19 \\
12,26\end{array}$ & $\begin{array}{l}62 \\
26,9\end{array}$ \\
\hline Statistiques & $P$ & $<0,001$ & $<0,001$ & 0,017 & 0,003 & 0,006 & 0,015 & NS \\
\hline
\end{tabular}

$P$ est la valeur de probabilité obtenue par comparaison des valeurs avant et après intoxication par le test $t$ de Student; NS = non significatif au seuil de $5 \%$.

centrolobulaire, l'alcool allylique lèse en premier lieu la région périportale (Gopinath et Ford, 1972, Belinsky et al, 1986). Cette différence est la conséquence de l'hétérogénéité du lobule hépatique, mais, alors que le mécanisme de la nécrose centrolobulaire produite par le $\mathrm{CCl}_{4}$ est bien connu (production par le réticulum endoplasmique d'un métabolite actif), l'effet pathogène de l'alcool allylique reste encore l'objet de spéculations.

Les modifications des paramètres d'exploration fonctionnelle ont été comparées avec les résultats d'une étude réalisée avec le $\mathrm{CCl}_{4}$ dans des conditions semblables (intoxication aiguë sublétale induite par $0,8 \mathrm{ml} / \mathrm{kg}$ IM, de $\mathrm{CCl}_{4}$ mélangé à un volume égal d'huile de paraffine, Abbaoui, 1988). Ceci permet de noter, avec l'alcool allylique, les différences suivantes: i) effet plus marqué de réduction de l'élimination de la BSP, ii) accroissement plus important de la bilirubine conjuguée; cette augmentation ne résulte pas d'une stimulation des réactions de glucurono-conjugaison, mais plus sûrement d'un défaut d'élimination par l'hépatocyte de la bilirubine conjuguée, ou d'une choléstase intralobulaire induite par la lésion périportale. Ces modifications se rapprochent de celles décrites lors de l'intoxication par Lantana camara (Seawright, 1965).

Par comparaison avec l'intensité de l'élévation de la bilirubine, l'augmentation de l'ammoniémie est relativement faible, ce qui s'explique par le fait que l'uréogenèse est un processus très actif qui ne devient défaillant que dans le cas de lésions très étendues du parenchyme.

En conclusion, l'alcool allylique permet d'obtenir, chez le mouton, un modèle d'insuffisance hépatique par atteinte aiguë des régions périportales, différent dans sa physiopathologie de l'état d'insuffisance induit par le $\mathrm{CCl}_{4}$. Ce modèle peut être utilisé en complément d'autres insuffisances hépatiques chimio-induites pour évaluer l'influence des altérations du parenchyme hépatique sur la biotransformation des xénobiotiques.

Abbaoui A (1988) Thèse maîtrise sciences vétérinaires, École vétérinaire d'Alfort

Argaw T (1989) Thèse maîtrise sciences vétérinaires, École vétérinaire d'Alfort

Belinsky SA, Badr MZ, Kauffman FC, Thurman RG (1986) J Pharmacol Exp Ther $238,1132-1137$

Gopinath C, Ford EJH (1972) Vet Pathol 9, 99-108

Seawright AA (1965), Aust Vet J 41, 116-119 